\title{
The Cacophony of Interconnected Networks
}

\author{
V. H. P. Louzada, N. A. M. Araújo, J. S. Andrade Jr, and H. J. Herrmann
}

\begin{abstract}
The harmony of an orchestra emerges from the individual effort of musicians towards mutual synchronization of their tempi. When the orchestra is split between two concert halls communicating via Internet, a time delay is imposed which might hinder synchronization. We present this type of system as two interconnected networks of oscillators with a time delay and analyze its dynamics as a function of the couplings and communication lag. We describe a breathing synchronization regime, namely, for a wide range of parameters, two groups emerge in the orchestra within the same concert hall playing at different tempi. Each group has a mirror in the other hall, one group is in phase and the other in anti-phase with their mirrors. For strong couplings, a phase shift between halls might occur. The implications of our findings on other interconnected systems are also discussed.

Technology has furnished us with global connectivity changing the functioning of cooperative work, international business, and interpersonal relationships. Today, it is possible to distribute an orchestra over two concert halls in different continents. Fast Internet connections would provide the communication infrastructure to properly combine the sounds [12]. As there is always a physical limit speed to information transport, the communication between sub-orchestras is subjected to a time delay due to the distance between halls. As we discuss here, this time delay might pose a real challenge to the synchronizability among musicians, which is vital to
\end{abstract}

Vitor H. P. Louzada

Computational Physics, IfB, ETH Zurich, Wolfgang-Pauli-Strasse 27, 8093 Zurich, Switzerland. e-mail: louzada@ethz.ch

N. A. M. Araújo

Departamento de Física, Faculdade de Ciências, Universidade de Lisboa, P-1749-016 Lisboa, Portugal.

Centro de Física Teórica e Computacional, Universidade de Lisboa, Avenida Professor Gama Pinto 2, P-1649-003 Lisboa, Portugal.

Hans J. Herrmann · J. S. Andrade Jr Computational Physics, IfB, ETH Zurich, Wolfgang-Pauli-Strasse 27, 8093 Zurich, Switzerland. Departamento de Física, Universidade Federal do Ceará, 60451-970 Fortaleza, Ceará, Brazil 
a successful performance. When isolated, each sub-orchestra would naturally play in unison. However, in our model orchestra, when listening to the musicians in the other sub-orchestra, the musicians in the same hall can split into two groups, playing with different tempi, leading to breathing synchronization. Interestingly, the partner in the other hall will be always playing with the same tempo but either in phase or anti-phase, depending on their tempo.

Understanding the consequences of a communication lag is also of major concern in other fields $[13,7,16]$. The plasmodium Physarum polycephalum, an amoebalike organism consisting of a network of tubular structures for protoplasm flow, naturally shows periodic variations in its thickness, a useful skill when fighting predators. In a controlled experiment, two regions of the same organism have been physically separated by a certain distance with the possibility of fine tuning the communication between them $[32,33]$. Depending on the coupling strength and time delay, the regions have been shown to present phase and anti-phase synchronization of the oscillatory thickness. This is precisely what we show here for the orchestra in the regime of strong influence of other musicians in the same concert hall. In what follows, we focus on the example of the orchestra but the results might also have impact on several biological and techno-social systems as, for example, functional brain networks, living oscillators, or coupled power grids. For a more general discussion of these findings see Ref. [21].

When playing the same piece, musicians in an orchestra try to synchronize their tempi, i.e., they try to play the notes at the same pace. Thus, for example, one violinist focuses simultaneously on the tempi of instruments in the same hall and on the corresponding violin in the other hall. All the other musicians act in the same way. The synchronizability of this setup can then be discussed in the framework of interdependent networks of oscillators. Each concert hall is modeled by a network, where nodes are musicians and links represent the interaction between them. Additionally, each musician also establishes a special inter-network coupling with one partner playing the same instrument in the other network (concert hall). Intra- and inter-network couplings have different time scales: while the intra-network interactions can be considered instantaneous, the inter-network ones have a time delay that depends on the distance between concert halls. Recent geometrical studies of coupled networks with intra- and inter-network links have revealed novel features never observed for isolated networks [14]. In particular, it has been shown that the overall robustness is reduced [28] and the collapse of the system occurs through large cascades of failures $[9,8]$. Dynamic properties of coupled networks have also been studied [19, 35, 30, 23, 2, 10, 15].

The Kuramoto model is a usual approach to network synchronization [17, 25, $6,34,27,31,18,22,24,1,26,3,5,4,20]$. For illustration purposes, here we stay with the example of an orchestra. A population $\Theta$ of $n$ Kuramoto oscillators is considered to be mutually interacting, such as the musicians in one concert hall trying to keep the same tempo. We consider a random graph of average degree four. Each musician (oscillator) $i \in \Theta$ is described by a phase $\theta_{i}(t)$, representing her/his current position, and a natural tempo $\omega_{i}$, corresponding to the pre-defined tempo of the music. Since all musicians are playing the same piece, we assume 
$\omega_{i} \equiv \omega_{0}$. The actual tempo of a musician is defined as the time derivative of the phase, $\dot{\theta}_{i}(t)$. To play the piece harmoniously, musicians try to synchronize their tempi. This interaction can be modeled in terms of the Kuramoto model as $\dot{\theta}_{i}=$ $\omega_{0}+\sigma \sum_{j=1}^{n} A_{i j}^{\Theta} \sin \left(\theta_{j}-\theta_{i}\right)$, where the sum goes over all other musicians $(i \neq j)$, $\sigma$ is the coupling strength between them, and $\mathbf{A}^{\theta}$ is the connectivity matrix such that $A_{i j}^{\Theta}=1$ if musician $i$ is influenced by $j$ and zero otherwise. For simplicity, we assume that the musicians are all playing at the same unitary amplitude, so that the state of each musician can be described just by a phasor $e^{i \theta_{i}(t)}$.

The collective performance in one concert hall, namely, the synchronization of its network, is characterized here by the complex order parameter $r_{\Theta}(t) e^{i \Psi(t)}=\frac{1}{n} \sum_{j}^{n} e^{i \theta_{j}(t)}$, where the sum goes over all musicians, $\Psi(t)$ is the average phase, and the amplitude $0 \leq\left|r_{\Theta}(t)\right| \leq 1$ measures the global coherence, i.e., how synchronized the musicians are. If $r_{\Theta}(t)=1$ all musicians play the same note at the same time, while low values of $r_{\Theta}$ imply that a significant fraction of musicians are out of phase.

We introduce now a second population $\Gamma$, also of $n$ oscillators, representing the second concert hall. Within this network, musicians are coupled in the same way. Each $j \in \Gamma$ is coupled with the corresponding partner $i \in \Theta$, forming the internetwork couplings. In analogy to oscillators in $\Theta$, the motion of each oscillator is described by a phasor $e^{i \gamma_{j}(t)}$, of phase $\gamma_{j}(t)$. The inter-network coupling is subjected to a time delay $\tau$, corresponding to the time required for information to travel between concert halls [29]. Previous studies introduced time delay among oscillators of the same population [36,11]. Here we consider the competition between an instantaneous intra-network and a delayed inter-network coupling. In a nutshell, the performance of each musician is described by,

$$
\left\{\begin{array}{l}
\dot{\theta}_{i}=\omega_{0}+\sigma_{\mathrm{EX}} \sin \left(\gamma_{j(i)}^{t-\tau}-\theta_{i}\right)+\sigma_{\mathrm{IN}} \sum_{k=1}^{N} A_{i k}^{\Theta} \sin \left(\theta_{k}-\theta_{i}\right) \\
\dot{\gamma}_{j}=\omega_{0}+\sigma_{\mathrm{EX}} \sin \left(\theta_{i(j)}^{t-\tau}-\gamma_{j}\right)+\sigma_{\mathrm{IN}} \sum_{k=1}^{N} A_{i k}^{\Gamma} \sin \left(\gamma_{k}-\gamma_{j}\right)
\end{array}\right.
$$

where the superscript $t-\tau$ indicates the instant when the phases are calculated, and $\sigma_{\mathrm{EX}}$ and $\sigma_{\mathrm{IN}}$ are the inter and intra-network couplings, respectively. In music, frequency is typically related with the note. However, here we assume that the properties of the note are not relevant. Instead, the usual natural frequency of the Kuramoto model $(\omega)$ corresponds to the tempo and, therefore, frequency and tempo will be used as synonymous.

For two interconnected networks of oscillators with time delay, a weak intranetwork coupling, and random initial distribution of phases, two frequency communities emerge within the same network, each synchronized with its mirror in a breathing mode, as shown in Fig. 1(b) in which the position of each empty circle represents its phase. A frequency lock occurs within communities, which move in a cohesive fashion on the complex plane. Interestingly, pairs of nodes that are part of different networks oscillate with the same frequency but might be either in phase or anti-phase (phase shift of $\pi$ ). Consequently, the presence of these two frequency communities affects the measurement of the new global oscillatory state, 

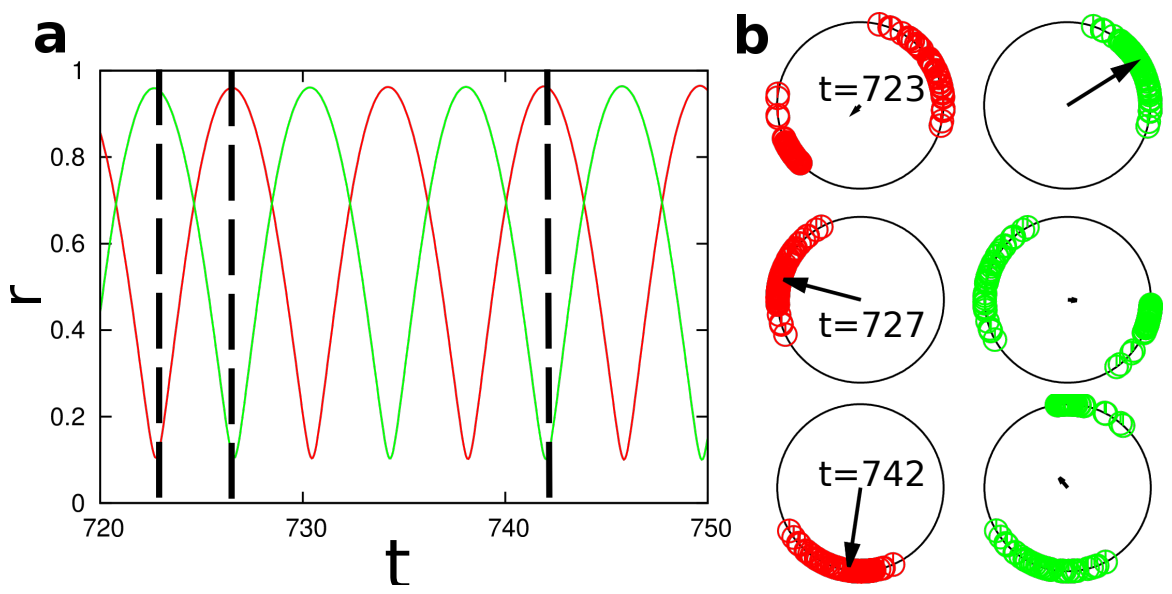

Fig. 1 The interactions between a strongly delayed inter-network coupling and a weak intranetwork coupling create two communities of different frequencies in steady state. a, The orderparameter of two populations (red and green) composed of $n=100$ oscillators each with $\omega_{0}=1.0$, $\tau=1.53, \sigma_{\mathrm{IN}}=0.01$, and $\sigma_{\mathrm{EX}}=0.5$. b. Snapshots of populations at three different time steps (black dashed vertical lines in a)), for the same parameters. Oscillators are spread across the complex plane according to their phase, with one plane for each network (left and right columns). The arrow at the center of each complex plane represents the order parameter of that network: its length representing the modulus and its argument representing the average phase. Superposition of the two groups of oscillators leads to breathing synchronization.

called breathing synchronization [21]. Figure 1(a) shows the time evolution of the order parameters $r_{\Theta}$ and $r_{\Gamma}$ for each population, quantifying this breathing behavior. For each curve, the maximum corresponds to the instant at which both groups of frequencies are in phase, while the minimum corresponds to an anti-phase between groups in the same network. Additionally, since for one frequency there is a phase shift of $\pi$ between inter-network pairs of nodes, the minimum in one network corresponds, necessarily, to the maximum in the other. In the context of the orchestra this implies that the communication lag in the interaction between concert halls generates a tendency to split musicians in each sub-orchestra into two groups playing at different tempi, hindering harmony. Moreover, cohesion within each community affects the amplitude of the breathing: the weaker the intra-network coupling, the smaller is this amplitude and more cacophony is present.

The observed breathing behavior is in deep contrast with what is expected for an isolated network $\left(\sigma_{\mathrm{EX}}=0\right)$. For isolated networks, the classical Kuramoto model is recovered, with frequency and phase synchronization emerging at a critical coupling $\sigma_{\mathrm{IN}}=\sigma_{\mathrm{IN}}^{*}$. Above this threshold, a macroscopic fraction of oscillators synchronizes with the same frequency and since here we consider the same natural frequency for all oscillators $\left(\omega_{i} \equiv \omega_{0}\right), \sigma_{\mathrm{IN}}^{*} \rightarrow 0$. The group of synchronized oscillators has frequency $\omega=\omega_{0}$ and the order parameter $r_{\Theta}(t)$ (or $r_{\Gamma}(t)$ ) saturates in time at a non-zero steady-state value, which is a monotonically increasing function of $\left(\sigma_{\mathrm{IN}}-\right.$ 


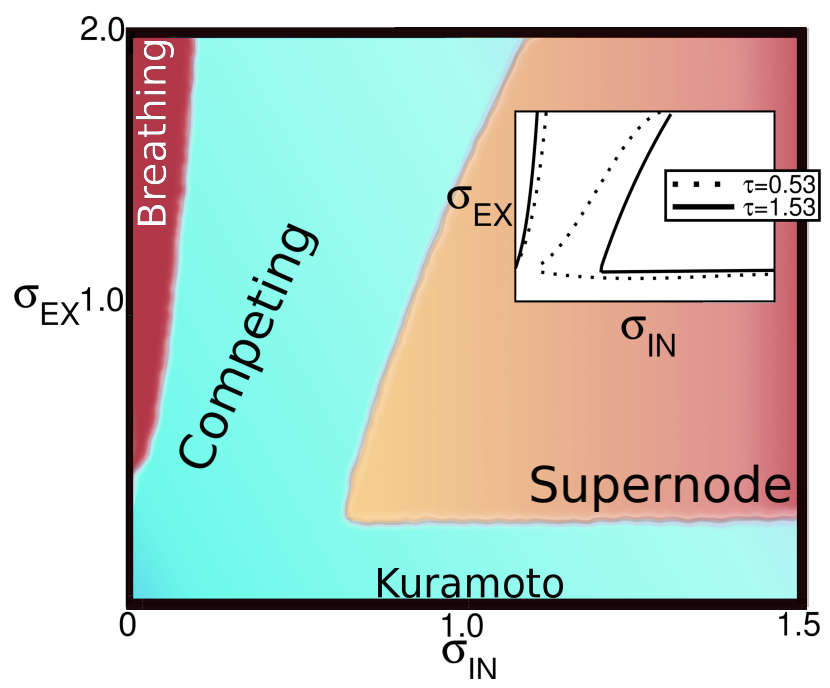

Fig. 2 Parameter space of two coupling strengths $\sigma_{\mathrm{ex}}$ and $\sigma_{\text {in }}$ showing that the prevalence of one frequency over the other changes according to the coupling strengths. The color of each area represents the number of stable frequencies: blue for one frequency, red for two. The inset exhibits the phase boundaries for different time delays. The dominant mechanisms of each region are labeled accordingly: breathing, (classical) kuramoto, competing, and supernode states.

$\sigma_{\text {IN }}^{*}$ ) [17]. It is worth noticing that in the case of coupled networks, and for sufficient inter-network couplings, none of the two frequencies is $\omega_{0}$.

Breathing synchronization is a direct consequence of the analytical solution obtained by Schuster and Wagner [29]. Depending on the initial phase difference between oscillators, the pair can synchronize with different frequencies $\omega$, which are solutions of,

$$
\omega=\omega_{0}-\sigma_{\mathrm{EX}} \sin (\omega \tau) .
$$

Notwithstanding of oscillating with the same frequency in the stationary state, the two oscillators might either be in phase, if $\cos (\omega \tau)>0$, or anti-phase. On interconnected networks, in the limit $\sigma_{\mathrm{IN}}=0$, the stationary state incorporates all conceivable solutions of Eq. 2. Surprisingly, our results with a weak coupling uncover two frequency groups with phase locking. In any case, the observed frequencies are consistent with the solution of Eq. 2.

To summarize the impact of several combinations of parameters, we plot in Fig. 2 the phase diagram in the space of the two coupling strengths $\left(\sigma_{\mathrm{IN}}\right.$ and $\left.\sigma_{\mathrm{EX}}\right)$. To recognize each regime, we compute the amount of oscillators with consistent steady frequency below and above the mean value of possible frequencies averaged over different samples. The color map of the main plot of Fig. 2 shows the ratio of these quantities. While the blue area represents the combinations of $\sigma_{\mathrm{IN}}$ and $\sigma_{\mathrm{EX}}$ that lead to a single frequency, the shades in red represent the two regions where two frequencies can be accomplished. Notice however that the two synchronization regimes in red differ in their underlying features. The one in the left (lower $\left.\sigma_{\mathrm{IN}}\right)$ is portrayed by 
the breathing behavior due to the presence of two frequency groups within each network. By contrast, in the supernode regime all nodes within a network are in phase locking, with the same frequency and, in this way, the order parameter is steady in time at this state. In the inset, we show the phase boundaries for distinctive time delays from which one can similarly see that the transition between regimes changes considerably.

The presence of a time delay between two coupled networks of oscillators represents one more test to the worldwide control of an orchestra, or any other global system. We have shown that the interchange between coupling and delay leads to states of either one unique or two conceivable synchronized frequencies. We have discovered that, even with a weak intra-network coupling, oscillators inside the same network split into two frequency groups, each group mirroring the other in the opposite network by oscillating at the same frequency. However, contingent upon their frequency, a group can be either in phase or anti-phase with its mirror in the other network, resulting in breathing synchronization. Additionally, we show that an arbitrary increment of the intra-network coupling is not an option to achieve phase and frequency synchronization regardless of its starting conditions. In a certain region of the parameter space, the intra-network coupling promotes the formation of two supernodes (one for every network), and two frequencies become stable.

It is safe to say that we are some years away of having a working setup of an intercontinental orchestra. In addition to all the technical challenges, our study shows that the very nature of a time-delayed system imposes a threat to the synchronization of the musicians. It would be rather interesting in fact to observe oscillations with two different frequencies within the musicians on the same continent due to the communication lag with the other region, resulting in a breathing synchronization cacophony.

Acknowledgements Authors would like to thank the Swiss National Science Foundation under contract 200021 126853, the CNPq, Conselho Nacional de Desenvolvimento Científico e Tecnológico - Brasil, the CNPq/FUNCAP Pronex grant, the ETH Zurich Risk Center, and the INCTSC-Brasil for financial support. This work was also supported by grant number FP7-319968 of the European Research Council. NA acknowledges financial support from the Portuguese Foundation for Science and Technology (FCT) under Contract no. IF/00255/2013.

\section{References}

1. Acebrón, J.A., Bonilla, L.L., Vicente, C.J.P., Ritort, F., Spigler, R.: The Kuramoto model : A simple paradigm for synchronization phenomena. Rev Mod Phys 77, 137-185 (2005)

2. Araújo, N.A.M., Seybold, H., Baram, R.M., Herrmann, H.J., Andrade Jr, J.S.: Optimal Synchronizability of Bearings. Phys Rev Lett 110, 064,106 (2013)

3. Arenas, A., Díaz-Guilera, A., Kurths, J., Moreno, Y., Zhou, C.: Synchronization in complex networks. Phys Rep 469, 93-153 (2008)

4. Barrat, A., Barthelemy, M., Vespignani, A.: Dynamical Processes on Complex Networks. Cambridge Univ. Press, Cambridge (2008)

5. Boccaletti, S.: The synchronized dynamics of complex systems. Elsevier, Amsterdam (2008) 
6. Boccaletti, S., Kurths, J., Osipov, G., Valladares, D.L., Zhou, C.S.: The synchronization of chaotic systems. Phys Rep 366, 1-101 (2002)

7. Boccaletti, S., Latora, V., Moreno, Y., Chavez, M., Hwang, D.: Complex networks: Structure and dynamics. Phys Rep 424, 175-308 (2006)

8. Brummitt, C.D., D’Souza, R.M., Leicht, E.A.: Suppressing cascades of load in interdependent networks. P Natl Acad Sci USA 109, E680-E689 (2011)

9. Buldyrev, S.V., Parshani, R., Paul, G., Stanley, H.E., Havlin, S.: Catastrophic cascade of failures in interdependent networks. Nature 464, 1025-1028 (2010)

10. Cardillo, A., Gómez-Gardeñes, J., Zanin, M., Romance, M., Papo, D., Pozo, F.D., Boccaletti, S.: Emergence of network features from multiplexity. Scientific Reports 3, 1344 (2013)

11. Choi, M.Y., Kim, H.J., Kim, D., Hong, H.: Synchronization in a system of globally coupled oscillators with time delay. Phys Rev E 61, 371-381 (2000)

12. Driessen, P.F., Darcie, T.E., Pillay, B.: The Effects of Network Delay on Tempo in Musical Performance. Comput. Music J. 35, 76-89 (2011)

13. Duke, C.: Prosperity, complexity and science. Nat Phys 2, 426-428 (2006)

14. Gao, J., Buldyrev, S.V., Stanley, H.E., Havlin, S.: Networks formed from interdependent networks. Nat Phys 8, 40-48 (2011)

15. Gómez, S., Díaz-Guilera, A., Gómez-Gardeñes, J., Pérez-Vicente, C.J., Moreno, Y., Arenas, A.: Diffusion Dynamics on Multiplex Networks. Phys Rev Lett 110, 028,701 (2013)

16. Helbing, D.: Systemic Risks in Society and Economics. Paper prepared for IRGC Workshop on Emerging Risks (2009)

17. Kuramoto, Y., Nishikawa, I.: Statistical Macrodynamics of Large Dynamical Systems. Case of a Phase Transition in Oscillator Communities. J Stat Phys 49, 569-605 (1987)

18. Li, C., Chen, G.: Synchronization in general complex dynamical networks with coupling delays. Physica A 343, 263-278 (2004)

19. Li, C., Sun, W., Kurths, J.: Synchronization between two coupled complex networks. Phys Rev E 76, 046,204 (2007)

20. Louzada, V.H.P., Araújo, N.A.M., Andrade Jr, J.S., Herrmann, H.J.: How to suppress undesired synchronization. Scientific Reports 2, 658 (2012). DOI 10.1038/srep00658

21. Louzada, V.H.P., Araújo, N.A.M., Andrade Jr, J.S., Herrmann, H.J.: Breathing synchronization in interconnected networks. Scientific Reports 3, 3289 (2013)

22. Lü, J., Chen, G.: A Time-Varying Complex Dynamical Network Model and Its Controlled Synchronization Criteria. IEEE T Automat Contr 50, 841-846 (2005)

23. Mao, X.: Stability switches, bifurcation, and multi-stability of coupled networks with time delays. Appl Math Comput 218, 6263-6274 (2012)

24. Motter, A.E., Zhou, C.S., Kurths, J.: Enhancing complex-network synchronization. Europhys Lett 69, 334-340 (2005)

25. Néda, Z., Ravasz, E., Vicsek, T., Brechet, Y., Barabási, A.: Physics of the rhythmic applause. Phys Rev E 61, 6987-6992 (2000)

26. Osipov, G., Kurths, J., Zhou, C.: Synchronization in oscillatory networks. Springer Verlag, New York (2007)

27. Pikovsky, A., Rosenblum, M., Kurths, J.: Synchronization: A universal concept in nonlinear sciences, Vol. 12. Cambridge Univ. Press, Cambridge (2003)

28. Schneider, C.M., Moreira, A.A., Andrade Jr, J.S., Havlin, S., Herrmann, H.J.: Mitigation of malicious attacks on networks. P Natl Acad Sci USA 108, 3838-3841 (2011)

29. Schuster, H.G., Wagner, P.: Mutual Entrainment of Two Limit Cycle Oscillators with Time Delayed Coupling. Prog Theor Phys 81, 939-945 (1989)

30. Shang, Y., Chen, M., Kurths, J.: Generalized synchronization of complex networks. Phys Rev E 80, 027,201 (2009)

31. Strogatz, S.H.: The Emerging Science of Spontaneous Order. Hyperion, New York (2003)

32. Takamatsu, A., Fujii, T., Endo, I.: Time delay effect in a living coupled oscillator system with the plasmodium of Physarum polycephalum. Phys Rev Lett 85, 2026 (2000)

33. Takamatsu, A., Takaba, E., Takizawa, G.: Environment-dependent morphology in plasmodium of true slime mold Physarum polycephalum and a network growth model. J Theor Biol 256, 29-44 (2009) 
34. Wang, X.F.: Complex Networks: Topology, Dynamics and Synchronization. Int J Bifucart Chaos 12, 885-916 (2002)

35. Wu, X., Zheng, W.X., Zhou, J.: Generalized outer synchronization between complex dynamical networks. Chaos 19, 013,109 (2009)

36. Yeung, M.K., Strogatz, S.H.: Time Delay in the Kuramoto Model of Coupled Oscillators. Phys Rev Lett 82, 648 (1999) 